\title{
Egg consumption and risk of incident type 2 diabetes: a dose-response meta-analysis of prospective cohort studies
}

\author{
Martha Tamez ${ }^{1}$, Jyrki K. Virtanen ${ }^{2}$ and Martin Lajous ${ }^{3,4 *}$ \\ ${ }^{1}$ Department of Nutrition, Harvard T. H. Chan School of Public Health, 677 Huntington Avenue, Boston, MA 02115, USA \\ ${ }^{2}$ Institute of Public Health and Clinical Nutrition, University of Eastern Finland, PO Box 1627, 70211 Kuopio, Finland \\ ${ }^{3}$ Center for Research on Population Health, National Institute of Public Health (Instituto Nacional de Salud Pública), \\ Av Universidad 655, Santa María Abuacatitlán, 62100 Cuernavaca, Mexico \\ ${ }^{4}$ Department of Global Health and Population, Harvard T. H. Chan School of Public Health, 677 Huntington Avenue, Boston, \\ MA 02115, USA
}

(Submitted 6 October 2015 - Final revision received 28 February 2016 - Accepted 11 March 2016 - First published online 25 April 2016)

\begin{abstract}
Experimental data suggest that egg intake could have a beneficial impact on several risk factors for type 2 diabetes. In contrast, some recent epidemiological studies have concluded that egg consumption may increase diabetes risk. We performed a dose-response meta-analysis of prospective cohorts on the relation of egg consumption with incident type 2 diabetes. We searched for cohort studies that assessed egg consumption and diabetes risk up to June 2015. We identified 416 articles and extracted data independently and in duplicate from ten eligible studies. We used random-effects generalised least squares models for pooled dose-response estimation based on thirteen estimates. Our study included 251213 individuals and 12156 incident type 2 diabetes cases. Egg intake was associated with incident type 2 diabetes (risk ratio (RR)/egg per d 1.13; $95 \%$ CI 1.04, 1.22). We identified study location as a major source of heterogeneity. For studies conducted in the USA, we observed a stronger association (RR 1.47; $95 \%$ CI 1.32, 1.64), whereas results were null for studies conducted elsewhere. Studies considered to be of high quality yielded null findings (RR 0.94; $95 \%$ CI $0 \cdot 74,1 \cdot 19$ ). The association of egg intake with increased risk of incident type 2 diabetes may be restricted to US cohort studies. There are limited data to support a biological mechanism that could underlie this association; thus, the possibility that these results may be due to residual confounding by dietary behaviours restricted to certain populations cannot be excluded.
\end{abstract}

Key words: Diets: Nutrition: Diabetes mellitus: Cohorts

In observational studies, cholesterol intake has been associated with impaired glucose metabolism ${ }^{(1)}$ and type 2 diabetes risk $^{(2,3)}$. Preliminary results from small randomised-controlled trials have shown that adding eggs, an important source of dietary cholesterol, to the diet improved insulin sensitivity ${ }^{(4)}$ and atherogenic lipoprotein profile ${ }^{(4,5)}$ and decreased inflammatory markers ${ }^{(6,7)}$. Furthermore, observational data support the role of circulating small and dense LDL and HDL particles ${ }^{(8)}$ and inflammation ${ }^{(9)}$ on diabetes risk. However, other nutrients found in eggs (e.g. choline) could also play a role in diabetes risk $^{(10-12)}$. In contrast, a previous meta-analysis of epidemiological studies concluded that egg consumption may increase diabetes risk $^{(13)}$. Owing to the absence of randomised trials directly assessing the effect of egg consumption on type 2 diabetes, we conducted a dose-response meta-analysis of prospective cohort studies to quantify the association between habitual egg intake and risk of type 2 diabetes.

\section{Methods}

\section{Search strategy}

We followed standard criteria for conducting and reporting meta-analyses of observational studies ${ }^{(14)}$. We searched for all prospective cohort studies that evaluated egg consumption and risk of diabetes mellitus in adults from the earliest available online indexing through June 2015. We conducted our study search without language restrictions on MEDLINE (egg[tw] or eggs[tiab] or 'animal food'[tiab]) and ('diabetes mellitus'[mesh] or diabetes[tiab]), EMBASE and EBSCOhost; we reviewed related articles, hand searched reference lists and directly contacted the authors. The search key words were 'eggs', 'animal food' and 'diabetes mellitus'. One investigator (M. T.) screened titles and abstracts, and two investigators (M. T., M. L.) independently and in duplicate reviewed full texts of potentially relevant articles and assessed study eligibility. We included

Abbreviation: RR, risk ratio.

* Corresponding author: M. Lajous, email mlajous@insp.mx 


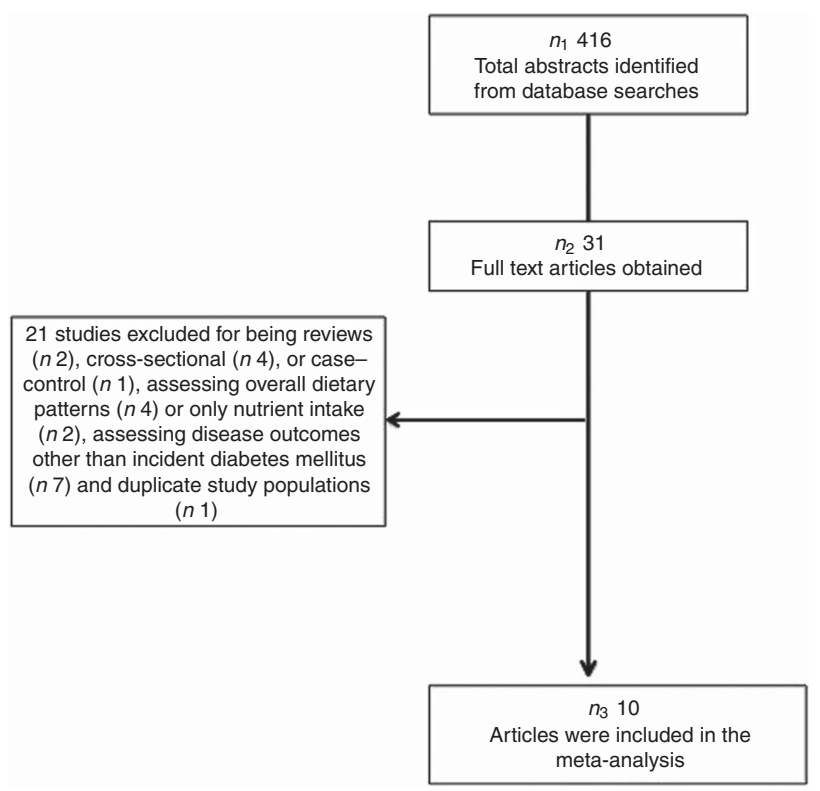

Fig. 1. Flow diagram of the study selection process.

studies that provided multivariable-adjusted risk estimates. We resolved differences by consensus. A priori we excluded ecological and cross-sectional analyses, case-control studies, commentaries, general reviews and case reports (Fig. 1). When duplicate studies were present, we chose the most recent publication. We also excluded studies reporting only crude risk estimates. After screening titles and abstracts of 416 articles and reviewing thirty-one full-texts, we extracted study characteristics and data in duplicate from ten eligible studies ${ }^{(15-24)}$ (Fig. 1) with a total of thirteen estimates. Thus, twenty-one studies were excluded because they were reviews $(n 2)^{(25,26)}$, cross-sectional $(n 4)^{(27-30)}$ or case-control $(n 1)^{(31)}$ studies; assessed overall dietary patterns

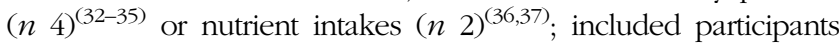
with prevalent disease $(n 4)^{(38-41)}$ or gestational diabetes $(n 1)^{(42)}$ or other outcome different from diabetes $(n 2)^{(43,44)}$; or because of duplicate study population $(n 1)^{(45)}$.

\section{Data extraction}

We extracted data using a standard electronic form independently and in duplicate by two investigators (M. T., M. L.). Information included the first author's name, contact information, year of publication, number of years the study was performed, study name, study location, population (age, sex, race, exclusion criteria and sample size), mean age and standard deviation at baseline, duration of follow-up, exposure assessment, egg consumption categories, outcome definition, outcome ascertainment, covariates adjusted for, number of participants, person-years, number of events and adjusted risk estimates and $95 \% \mathrm{CI}$. When the mean age of the total study population was unavailable, we calculated the weighted mean age and weighted standard deviation based on exposure categories' specific information. We assumed one serving of egg to be equivalent to $50 \mathrm{~g}$. We gave preference to multivariable estimates from models with the greatest control for potential confounders. Hazard ratios and OR were assumed to approximate risk ratios (RR). We assigned studies a degree of covariate adjustment: minimal (socio-demographic covariates), adequate (socio-demographic plus either other risk factors or dietary variables) and optimal (socio-demographic plus risk factors and dietary variables). Issues regarding missing data or definitions were resolved by direct contact with authors.

We assessed study quality based on the degree of covariate adjustment and the Newcastle-Ottawa quality assessment scale for observational studies in meta-analyses ${ }^{(46)}$. This scale for observational studies in meta-analyses grants a maximum of 9 points to each study: a maximum of 1 point for each item within the selection (representativeness of the exposed cohort, selection of the non-exposed cohort, ascertainment of exposure, demonstration that outcome of interest was not present at start of study) and outcome categories (assessment of outcome, length of follow-up, adequacy of follow-up) and a maximum of 2 points for the comparability category based on the design or analysis. We assigned scores of 0-6 for low-quality and 7-9 for high-quality studies based on the distribution of scores among studies. Differences in quality assessment scores between investigators were unusual and were resolved by consensus.

\section{Statistical analyses}

We conducted random-effects, dose-response regressions by using generalised least squares for trend estimation (one-stage estimation $)^{(47)}$. We assumed hazard ratios and OR-approximated RR. Covariance was fit with the use of total numbers of cases plus non-cases for studies that reported OR or person-years for studies that reported RR or hazard ratios, at each level of exposure. For completeness, we also performed two-stage estimation: separate generalised least squares models for trend were evaluated for each study to derive study-specific, log-linear dose-responses ( $\log \mathrm{RR}$ ), and then each study-specific $\log \mathrm{RR}$ was pooled in a second generalised least squares model for trend. We pre-specified one-stage estimation as our primary outcome because it uses all available estimates in each study yielding a better estimate of the variance-covariance matrix relative to the two-stage estimation. We tested the between-study heterogeneity with goodness of fit $\left(\chi^{2}\right)$. When exploring dietary factors in relation to diabetes, BMI is commonly included in models to adjust for confounders. However, this variable could also be considered an intermediate in the causal pathway. Therefore, we conducted sensitivity analyses including (when available) estimates from models that were not adjusted for BMI. We explored a priori potential sources of heterogeneity by using metaregression (sex, study location (USA $v$. Europe/Asia), study quality (Newcastle-Ottawa score $<7$ or $\geq 7$ ), years of follow-up $(<15, \geq 15)$, mean age $(<50, \geq 50$ years) and method for assessing dietary intakes (FFQ $v$. other methods)). We constructed funnel plots for visual inspection of publication bias and evaluated statistically the bias using Begg's test ${ }^{(48)}$. Finally, we stratified the data by sex, study location and quality score. Analyses were performed using Stata 11.2 for Mac (StataCorp LP), with twotailed $\alpha<0.05$. Analytical code used is provided in the online 
Supplementary Material, and databases and documentation are available as supplemental digital content.

\section{Results}

We identified ten studies with thirteen different estimates that included 251213 individuals (173463 women and 77750 men) and 12156 incident cases of type 2 diabetes. The studies were conducted in the USA ( $n$ 4), Asia ( $n$ 1) and Europe $(n 5)$. Age ranged from 38 to 95 years, and the median daily egg consumption ranged from 0 to $1 \cdot 1$ eggs across studies (Table 1 ). Nearly all studies adequately adjusted for important diabetes risk factors including age, sex, BMI, smoking status, alcohol use, physical activity and dietary factors.

Each egg per day was associated with a $13 \%$ higher risk of incident type 2 diabetes (one-stage estimation RR 1.13; 95\% CI 1.04, 1.22; $P_{\text {heterogeneity }}<0.001$ ) (Fig. 2). In contrast, secondary results from the two-stage estimation (which does not use all available information) were null (RR 1.07; 95\% CI 0.93, 1.24). The results from a sensitivity analysis that used estimates that did not include BMI, a potential intermediate, were qualitatively the same (online Supplementary Fig. S1). Using meta-regression, we explored sources of heterogeneity: sex $(P=0.84)$, mean age $(P=0 \cdot 15)$, study location $(P=0.03)$, study quality $(P=0 \cdot 18)$, years of follow-up $(P=0 \cdot 46)$ and method for assessing dietary intakes $(P=0 \cdot 20)$. When we stratified the analyses by study location, we observed that in the studies conducted in the USA an egg per day was associated with a $47 \%$ higher risk of type 2 diabetes (RR 1.47; 95\% CI 1.32, 1.64), whereas the association for studies conducted elsewhere was null (Table 2). Moreover, the association for high-quality studies was null (RR 0.94; 95\% CI 0.74, 1.19). We found no evidence of publication bias on the funnel plot or Begg's test $(P=0 \cdot 46)$ (Fig. 3).

\section{Discussion}

In a dose-response meta-analysis of ten publications of prospective studies using thirteen estimates, we observed a direct association between egg consumption and type 2 diabetes. We found evidence that results may be driven in part by studies conducted in the USA and by studies of a lower quality.

In animal studies, cholesterol intake has been associated with impaired glucose metabolism ${ }^{(49)}$ and inflammation ${ }^{(50)}$. However, these studies generally use a very high dose of cholesterol, thus potentially limiting the applicability of results to humans. Eggs are important contributors of dietary cholesterol, raising concerns that egg consumption may affect cardiovascular health and diabetes risk. However, there is no clear relationship between dietary cholesterol consumption and serum cholesterol ${ }^{(39)}$, although there seems to be significant heterogeneity in the response to cholesterol intake. In addition to genetic factors ${ }^{(51)}$, for example, obesity and insulin resistance appear to affect cholesterol absorption ${ }^{(52,53)}$. In addition, experimental studies in humans have shown that increased egg intake has rather had a beneficial impact on several risk factors for type 2 diabetes, such as insulin resistance $e^{(4)}$, inflammation ${ }^{(6,7,54)}$ and lipid particle size $e^{(4,5)}$. However, a previous meta-analysis of prospective cohorts concluded that individuals who ate an egg or more per day had a $42 \%$ higher risk of diabetes compared with individuals who never consumed eggs ${ }^{(13)}$. This meta-analysis was based on five cohorts, included US studies only and dose-response was not assessed ${ }^{(16-18)}$.

Contrasting results from intervention studies and observational studies should take into account design limitations. Conducting randomised trials to evaluate the effects of foods can be challenging because of costs, difficulties in blinding individuals and non-compliance because of the length of time that is necessary to observe incidence of outcomes. For example, only short-term intervention studies on egg consumption using intermediate risk markers are feasible but do not necessarily reflect diabetes risk. Thus, in the absence of trials that use diabetes as the outcome, one strategy is to infer these effects from long-term prospective cohort studies. Besides the fact that these studies may not adequately reflect the question of the potential effect of altering food consumption, two key limitations are the potential for residual confounding and for misclassification of the exposure. Our results are based on individual studies where the potential for these two major limitations is always present, and therefore the results should be interpreted with caution.

The observation that the association could be driven by studies conducted in the USA may reflect the possibility that egg consumption may be confounded by behaviours or dietary habits associated with diabetes risk that are common in this population. For example, in the US studies, egg intake is often associated with smoking ${ }^{(17,18,21)}$ or lower physical activity ${ }^{(17)}$ or higher intake of red meat ${ }^{(17,18,21)}$, whereas this is generally not observed in studies outside the USA ${ }^{(19,24)}$. However, although one study in France did find such associations with egg intake, it still reported null findings for the relation of egg intake and type 2 diabetes ${ }^{(23)}$. Food preparation methods (e.g. boiled or fried eggs, whole eggs or only egg whites) or concurrent consumption of other foods that may increase diabetes risk (e.g. home fries, bacon) may also account for a part of the differences, but such information is not available in these studies. Our results are consistent with a recently published meta-analysis ${ }^{(55)}$, and the conclusion that the association was not present in non-US populations is strengthened by the inclusion in the current meta-analysis of an additional study from Finland. The importance of adequate designs, robust ascertainment of exposure and outcome and collection of information on potential confounding factors with as much detail as possible is further underscored by the observation that better quality studies were less likely to find an association between egg consumption and diabetes risk. We classified four studies as low quality mainly because diabetes was self-reported and the follow-up rate was inadequate or not described $^{(16,17,20)}$. It is difficult to interpret why results of these studies differ from high-quality studies. However, three of these studies were conducted in the USA.

The possibility that the observed differences across populations are the result of underlying biological mechanisms is still present. Intestinal microbiota may vary across populations and there is evidence that intestinal flora affects the production of trimethylamine-N-oxide from dietary phosphatidylcholine 


\section{N British Journal of Nutrition}

Table 1. Characteristics of participants in included studies of egg consumption and type 2 diabetes risk

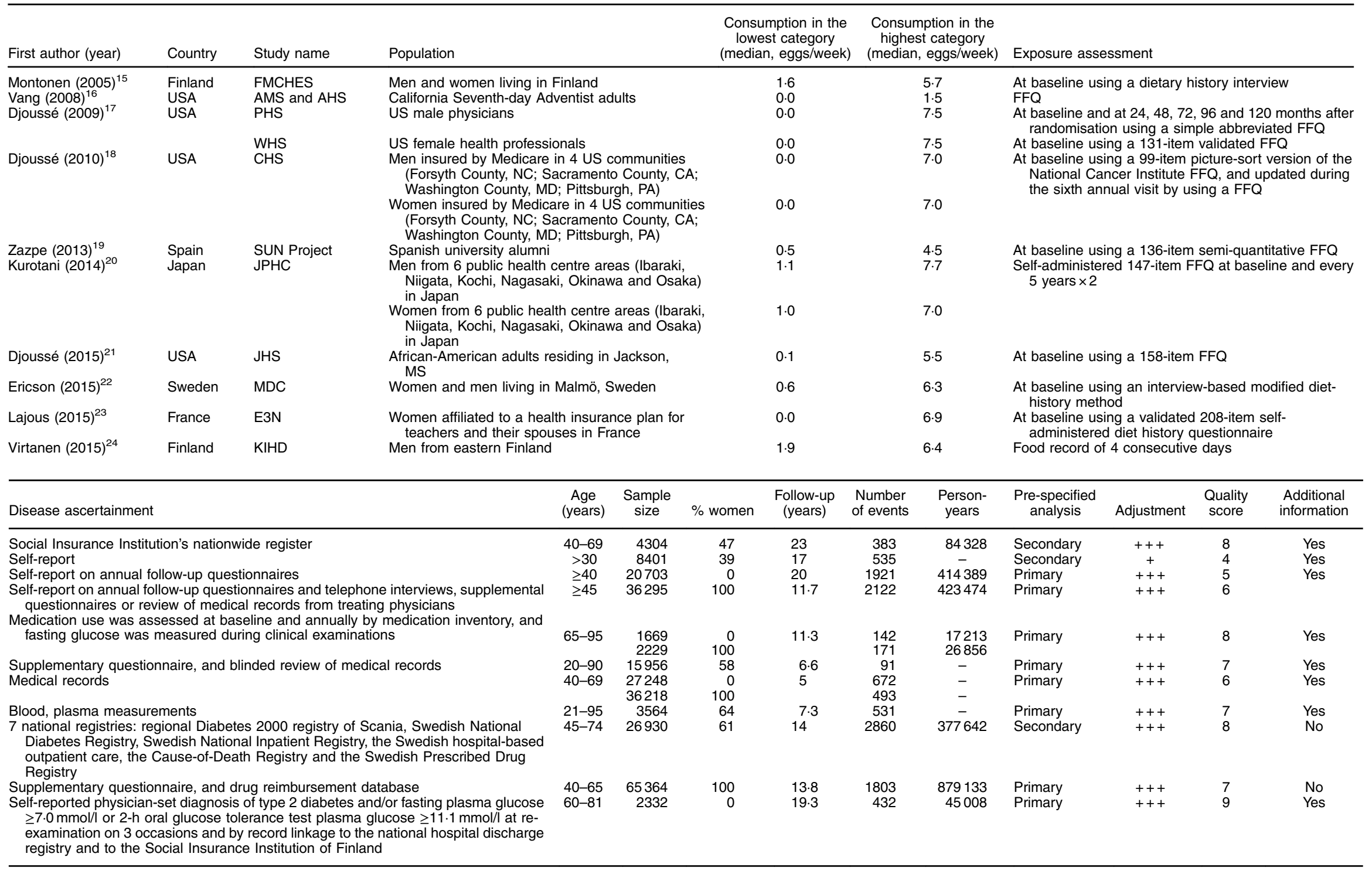

FMCHES, Finnish Mobile Clinic Health Examination Survey; AMS, Adventist Mortality Study; AHS, Adventist Health Study; PHS, Physicians' Health Study; WHS, Women's Health Study; CHS, Cardiovascular Health Study; SUN, Seguimiento Universidad de Navarra; JPHC, Japan Public Health Center-based Prospective; JHS, Jackson Heart Study; MDC, Malmö Diet and Cancer Cohort; E3N, Etude Epidémiologique auprès des femmes de la Mutuelle Générale de l'Education Nationale; KIHD, Kuopio Ischaemic Heart Disease Risk Factor Study. 


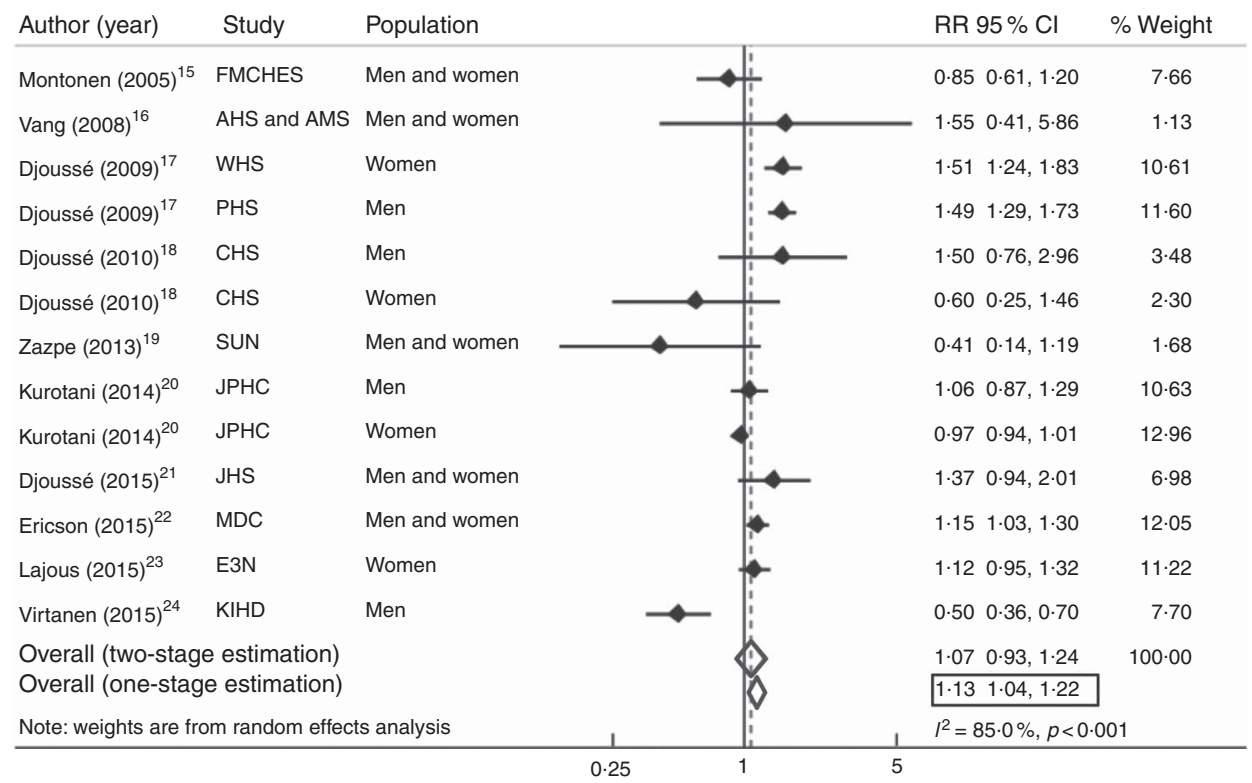

RR of type 2 diabetes per egg per day

Fig. 2. Risk ratios (RR) of incident type 2 diabetes per egg per day. One-stage overall pooled dose-response estimate and $95 \% \mathrm{Cl}$ are boxed. $\rightarrow$ and Study-specific dose-response and $95 \% \mathrm{Cl}$; - - - -, pooled dose-response; $\diamond, 95 \% \mathrm{Cl}$ combining each study specific dose-response (two-stage); FMCHES, Finnish Mobile Clinic Health Examination Survey; AHS, Adventist Health Study; AMS, Adventist Mortality Study; WHS, Women's Health Study; PHS, Physicians' Health Study; CHS, Cardiovascular Health Study; SUN, Seguimiento Universidad de Navarra; JPHC, Japan Public Health Center-based Prospective; JHS, Jackson Heart Study; MDC, Malmö Diet and Cancer Cohort; E3N, Etude Epidémiologique auprès des femmes de la Mutuelle Générale de l'Education Nationale; KIHD, Kuopio Ischemic Heart Disease Risk Factor Study.

Table 2. Consumption and risk of type 2 diabetes stratified by sex, study location and study quality (Risk ratios (RR) and $95 \%$ confidence intervals)

\begin{tabular}{|c|c|c|c|c|c|}
\hline Subgroup analyses & Number of estimates & $\mathrm{RR}$ & $95 \% \mathrm{Cl}$ & $P_{\text {heterogeneity }}$ & $P^{2}(\%)$ \\
\hline \multicolumn{6}{|l|}{ Sex } \\
\hline Men & 4 & 1.03 & $0.66,1.61$ & $P<0.001$ & $91 \cdot 7$ \\
\hline Women & 4 & 1.12 & $0.88,1.41$ & $P<0.001$ & $86 \cdot 3$ \\
\hline Men and women & 5 & 1.07 & $0.84,1.36$ & $P=0.12$ & $45 \cdot 6$ \\
\hline \multicolumn{6}{|l|}{ Study location } \\
\hline USA & 6 & 1.47 & $1.32,1.64$ & $P=0.53$ & 0 \\
\hline Asia/Europe & 7 & 0.95 & $0.83,1.10$ & $P<0.001$ & $79 \cdot 1$ \\
\hline \multicolumn{6}{|l|}{ Study quality score } \\
\hline Low $(0-6)$ & 5 & 1.24 & $0.96,1.59$ & $P<0.001$ & $91 \cdot 8$ \\
\hline $\operatorname{High}(7-9)$ & 8 & 0.94 & $0.74,1.19$ & $P<0.001$ & $76 \cdot 7$ \\
\hline
\end{tabular}

(egg yolks are important contributors) ${ }^{(10)}$. In animal studies, this metabolite appears to play a role in glucose metabolism ${ }^{(12)}$. Thus, there is a need to further study this association between egg consumption and glucose metabolism across populations.

Our meta-analysis has several strengths. We systematically reviewed multiple databases for all prospective studies on egg consumption and diabetes risk. We contacted authors directly when clarifications of findings or additional data were necessary, thus minimising potential misclassification and publication bias. We performed study inclusion/exclusion and data extraction in duplicate and independently. We explicitly assessed dose-response rather than carrying out simple categorical comparisons using generalised least squares models for trend estimation.

Our results suggest that the association of habitual consumption of eggs and incidence of type 2 diabetes observed in

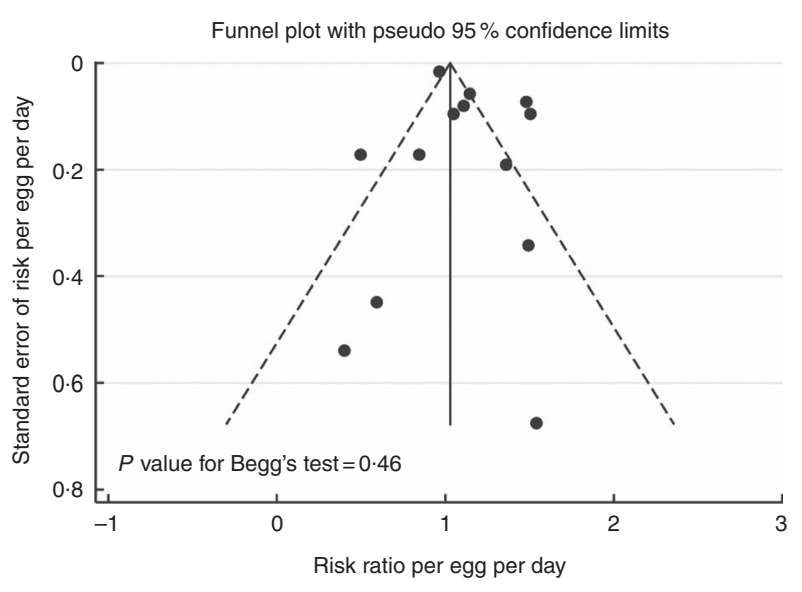

Fig. 3. Funnel plot for graphical assessment of potential publication bias. 
prospective studies may be restricted to studies conducted in the USA. In the absence of a clear biological mechanism, the possibility that the observed relation may be due to residual confounding by dietary behaviours or food preparation methods restricted to this population is always present.

\section{Acknowledgements}

The authors of the present study acknowledge the authors of studies included in this analysis who provided additional information.

This analysis was supported by the National Council of Science and Technology (CONACyT, Mexico), the University of Eastern Finland, National Institute of Public Health and the Bernard Lown Fund for Cardiovascular Health.

M. L. and J. K. V. conceived the study. M. L. and M. T. extracted data and contacted authors. M. T. conducted the analysis. M. L., M. T. and J. K. V. wrote and revised the manuscript.

M. L. has an investigator-initiated research grant from AstraZeneca. Other authors have no disclosures.

\section{Supplementary material}

For supplementary material/s referred to in this article, please visit http://dx.doi.org/doi:10.1017/S000711451600146X

\section{References}

1. Feskens EJ \& Kromhout D (1990) Habitual dietary intake and glucose tolerance in euglycaemic men: the Zutphen Study. Int J Epidemiol 19, 953-959.

2. Meyer KA, Kushi LH, Jacobs DR Jr, et al. (2001) Dietary fat and incidence of type 2 diabetes in older Iowa women. Diabetes Care 24, 1528-1535.

3. Salmeron J, Hu FB, Manson JE, et al. (2001) Dietary fat intake and risk of type 2 diabetes in women. Am J Clin Nutr 73, 1019-1026.

4. Blesso CN, Andersen CJ, Barona J, et al. (2013) Whole egg consumption improves lipoprotein profiles and insulin sensitivity to a greater extent than yolk-free egg substitute in individuals with metabolic syndrome. Metabolism 62, 400-410.

5. Mutungi G, Waters D, Ratliff J, et al. (2010) Eggs distinctly modulate plasma carotenoid and lipoprotein subclasses in adult men following a carbohydrate-restricted diet. $J$ Nutr Biochem 21, 261-267.

6. Ratliff JC, Mutungi G, Puglisi MJ, et al. (2008) Eggs modulate the inflammatory response to carbohydrate restricted diets in overweight men. Nutr Metab (Lond) 5, 6.

7. Andersen CJ, Lee JY, Blesso CN, et al. (2014) Egg intake during carbohydrate restriction alters peripheral blood mononuclear cell inflammation and cholesterol homeostasis in metabolic syndrome. Nutrients 6, 2650-2667.

8. Hodge AM, Jenkins AJ, English DR, et al. (2009) NMR-determined lipoprotein subclass profile predicts type 2 diabetes. Diabetes Res Clin Pract 83, 132-139.

9. Wang X, Bao W, Liu J, et al. (2013) Inflammatory markers and risk of type 2 diabetes: a systematic review and meta-analysis. Diabetes Care 36, 166-175.

10. Tang WH, Wang Z, Levison BS, et al. (2013) Intestinal microbial metabolism of phosphatidylcholine and cardiovascular risk. N Engl J Med 368, 1575-1584.
11. Lever M, George PM, Slow S, et al. (2014) Betaine and trimethylamine-N-oxide as predictors of cardiovascular outcomes show different patterns in diabetes mellitus: an observational study. PLOS ONE 9, e114969.

12. Gao X, Xu J, Jiang C, et al. (2015) Fish oil ameliorates trimethylamine N-oxide-exacerbated glucose intolerance in high-fat diet-fed mice. Food Funct 6, 1117-1125.

13. Shin JY, Xun P, Nakamura Y, et al. (2013) Egg consumption in relation to risk of cardiovascular disease and diabetes: a systematic review and meta-analysis. Am J Clin Nutr $\mathbf{9 8 ,}$ 146-159.

14. Stroup DF, Berlin JA, Morton SC, et al. (2000) Meta-analysis of observational studies in epidemiology: a proposal for reporting. Meta-analysis Of Observational Studies in Epidemiology (MOOSE) group. JAMA 283, 2008-2012.

15. Montonen J, Jarvinen R, Heliovaara M, et al. (2005) Food consumption and the incidence of type II diabetes mellitus. Eur J Clin Nutr 59, 441-448.

16. Vang A, Singh PN, Lee JW, et al. (2008) Meats, processed meats, obesity, weight gain and occurrence of diabetes among adults: findings from Adventist Health Studies. Ann Nutr Metab 52, 96-104.

17. Djoussé L, Gaziano JM, Buring JE, et al. (2009) Egg consumption and risk of type 2 diabetes in men and women. Diabetes Care 32, 295-300.

18. Djoussé L, Kamineni A, Nelson TL, et al. (2010) Egg consumption and risk of type 2 diabetes in older adults. Am J Clin Nutr 92, 422-427.

19. Zazpe I, Beunza JJ, Bes-Rastrollo M, et al. (2013) Egg consumption and risk of type 2 diabetes in a Mediterranean cohort; the sun project. Nutr Hosp 28, 105-111.

20. Kurotani K, Nanri A, Goto A, et al. (2014) Cholesterol and egg intakes and the risk of type 2 diabetes: the Japan Public Health Center-based Prospective Study. Br J Nutr 112, 1636-1643.

21. Djoussé L, Petrone AB, Hickson DA, et al. (2015) Egg consumption and risk of type 2 diabetes among African Americans: the Jackson Heart Study. Clin Nutr 103, 474-480.

22. Ericson U, Hellstrand S, Brunkwall L, et al. (2015) Food sources of fat may clarify the inconsistent role of dietary fat intake for incidence of type 2 diabetes. Am J Clin Nutr 101, $1065-1080$

23. Lajous M, Bijon A, Fagherazzi G, et al. (2015) Egg and cholesterol intake and incident type 2 diabetes among French women. Br J Nutr 114, 1667-1673.

24. Virtanen JK, Mursu J, Tuomainen TP, et al. (2015) Egg consumption and risk of incident type 2 diabetes in men: the Kuopio Ischaemic Heart Disease Risk Factor Study. Am J Clin Nutr 101, 1088-1096.

25. Jarrett RJ (1984) Type 2 (non-insulin-dependent) diabetes mellitus and coronary heart disease-chicken, egg or neither? Diabetologia 26, 99-102.

26. Djoussé L (2013) Relation of eggs with incident cardiovascular disease and diabetes: friends or foes? Atherosclerosis $\mathbf{2 2 9}$, 507-508.

27. Agrawal S \& Ebrahim S (2012) Prevalence and risk factors for self-reported diabetes among adult men and women in India: findings from a national cross-sectional survey. Public Health Nutr 15, 1065-1077.

28. Kim HS, Park SY, Grandinetti A, et al. (2008) Major dietary patterns, ethnicity, and prevalence of type 2 diabetes in rural Hawaii. Nutrition 24, 1065-1072.

29. Wang CN, Liang Z, Wei P, et al. (2002) Changes in dietary patterns and certain nutrition-related diseases in urban and rural residents of Jiangsu Province, China, during the 1990s. Biomed Environ Sci 15, 271-276. 
30. Shi Z, Yuan B, Zhang C, et al. (2011) Egg consumption and the risk of diabetes in adults, Jiangsu, China. Nutrition 27 194-198.

31. Radzeviciene L \& Ostrauskas R (2012) Egg consumption and the risk of type 2 diabetes mellitus: a case-control study. Public Health Nutr 15, 1437-1441.

32. Batis C, Mendez MA, Sotres-Alvarez D, et al. (2014) Dietary pattern trajectories during 15 years of follow-up and HbA1c, insulin resistance and diabetes prevalence among Chinese adults. J Epidemiol Community Health 68, 773-779.

33. Imamura F, Lichtenstein AH, Dallal GE, et al. (2009) Generalizability of dietary patterns associated with incidence of type 2 diabetes mellitus. Am J Clin Nutr 90, 1075-1083.

34. Liese AD, Weis KE, Schulz M, et al. (2009) Food intake patterns associated with incident type 2 diabetes: the Insulin Resistance Atherosclerosis Study. Diabetes Care 32, 263-268.

35. Yu D, Zhang X, Xiang YB, et al. (2014) Adherence to dietary guidelines and mortality: a report from prospective cohort studies of 134,000 Chinese adults in urban Shanghai. Am J Clin Nutr 100, 693-700.

36. Silva Ton WT, das Gracas de Almeida C, de Morais Cardoso L, et al. (2014) Effect of different protein types on second meal postprandial glycaemia in normal weight and normoglycemic subjects. Nutr Hosp 29, 553-558.

37. Houston DK, Ding J, Lee JS, et al. (2011) Dietary fat and cholesterol and risk of cardiovascular disease in older adults: the Health ABC Study. Nutr Metab Cardiovasc Dis 21, 430-437.

38. Pearce KL, Clifton PM \& Noakes M (2011) Egg consumption as part of an energy-restricted high-protein diet improves blood lipid and blood glucose profiles in individuals with type 2 diabetes. Br J Nutr 105, 584-592.

39. Fuller NR, Sainsbury A, Caterson ID, et al. (2015) Egg consumption and human cardio-metabolic health in people with and without diabetes. Nutrients 7, 7399-7420.

40. Gannon MC, Nuttall FQ, Lane JT, et al. (1992) Metabolic response to cottage cheese or egg white protein, with or without glucose, in type II diabetic subjects. Metabolism 41, $1137-1145$.

41. Horwath CC \& Worsley A (1991) Dietary habits of elderly persons with diabetes. J Am Diet Assoc 91, 553-557.

42. Qiu C, Frederick IO, Zhang C, et al. (2011) Risk of gestational diabetes mellitus in relation to maternal egg and cholesterol intake. Am J Epidemiol 173, 649-658.

43. Kuriki K, Tokudome S \& Tajima K (2004) Association between type II diabetes and colon cancer among Japanese with reference to changes in food intake. Asian Pac J Cancer Prev 5, 28-35.

44. Nicklas TA, O'Neil CE \& Fulgoni VL 3rd (2015) Differing statistical approaches affect the relation between egg consumption, adiposity, and cardiovascular risk factors in adults. $J$ Nutr 145, 170S-176S.

45. Ericson U, Sonestedt E, Gullberg B, et al. (2013) High intakes of protein and processed meat associate with increased incidence of type 2 diabetes. Br J Nutr 109, 1143-1153.

46. Wells GA, Shea B, O'Connell D, et al. (2011) The NewcastleOttawa Scale (NOS) for assessing the quality of nonrandomized studies in meta-analyses. http://www.ohri.ca/ programs/clinical_epidemiology/oxford.asp (accessed June 2015).

47. Greenland S \& Longnecker MP (1992) Methods for trend estimation from summarized dose-response data, with applications to meta-analysis. Am J Epidemiol 135, 1301-1309.

48. Begg CB \& Mazumdar M (1994) Operating characteristics of a rank correlation test for publication bias. Biometrics $\mathbf{5 0}$, 1088-1101.

49. Adamopoulos PN, Papamichael CM, Zampelas A, et al. (1996) Cholesterol and unsaturated fat diets influence lipid and glucose concentrations in rats. Comp Biochem Physiol B Biochem Mol Biol 113, 659-663.

50. Lewis KE, Kirk EA, McDonald TO, et al. (2004) Increase in serum amyloid a evoked by dietary cholesterol is associated with increased atherosclerosis in mice. Circulation 110, 540-545.

51. Ordovas JM, Lopez-Miranda J, Mata P, et al. (1995) Gene-diet interaction in determining plasma lipid response to dietary intervention. Atherosclerosis 118, Suppl., S11-S27.

52. Knopp RH, Retzlaff B, Fish B, et al. (2003) Effects of insulin resistance and obesity on lipoproteins and sensitivity to egg feeding. Arterioscler Thromb Vasc Biol 23, 1437-1443.

53. Tannock LR, O'Brien KD, Knopp RH, et al. (2005) Cholesterol feeding increases C-reactive protein and serum amyloid A levels in lean insulin-sensitive subjects. Circulation 111, 3058-3062.

54. Blesso CN, Andersen CJ, Barona J, et al. (2013) Effects of carbohydrate restriction and dietary cholesterol provided by eggs on clinical risk factors in metabolic syndrome. J Clin Lipidol 7, 463-471.

55. Djoussé L, Khawaja OA \& Gaziano JM (2016) Egg consumption and risk of type 2 diabetes: a meta-analysis of prospective studies. Am J Clin Nutr 103, 474-480. 